\title{
Protección de los trabajadores y trabajadoras de dirección, dentro de los supuestos establecidos en la ley orgánica del trabajo de la República Bolivariana de Venezuela
}

\author{
Protection of management workers, within the assumptions \\ established in the Organic Labor Law of the Bolivarian Republic of \\ Venezuela
}

\section{Proteção dos trabalhadores da gestão, dentro dos pressupostos estabelecidos na Lei Orgânica do Trabalho da República Bolivariana da Venezuela.}

Faridy Suarez Colmenares fary1702@gmail.com Circunscripción Judicial del Estado Carabobo, Venezuela

Articulo recibido en febrero 2018; arbitrado en marzo 2018; aprobado en abril 2018 y publicado junio de 2018

RESUMEN

El presente trabajo tiene como objetivo evaluar la exclusión o inclusión de los trabajadores y trabajadoras de dirección de la protección establecida en los artículos 420 ordinal 4 o, y 347 de la Ley Orgánica del Trabajo, las trabajadoras y los trabajadores, referidos a la protección por inamovilidad para los trabajadores con hijos con alguna discapacidad o enfermedad que les impida o dificulte valerse por sí mismos. Varios son los decretos presidenciales de inamovilidad en donde los trabajadores de dirección están excluidos del régimen de inamovilidad bien sea por decreto o por ley. Se utilizó la investigación documental, como metodología implementada. El propósito fue ampliar y profundizar el conocimiento de su naturaleza, con apoyo, principalmente, en fuentes bibliográficas y documentales. Se concluyó que extender los efectos de la inamovilidad a los trabajadores de dirección sería posible como protección a la familia y al niño con discapacidad y no propiamente a los padres, ya que los instrumentos legales vigentes, que promulgan el Sistema Rector Nacional para la Protección Integral del Niño, Niña y Adolescentes antes enunciados, dan cabida a la posibilidad de contar con esta protección.

Palabras clave: Inamovilidad; trabajadores de dirección; estabilidad; ley orgánica del trabajo
ABSTRACT

The objective of this paper is to evaluate the exclusion or inclusion of male and female managers in the protection established in articles 420 ordinal 4th, and 347 of the Organic Labor Law, workers, referred to the protection by irremovability for workers with children with a disability or illness that prevents or hinders them from working on their own. Several are the presidential decrees of irremovability in which management workers are excluded from the regime of irremovability either by decree or by law. Documentary research was used, as implemented methodology. The purpose was to expand and deepen the knowledge of its nature, with support, mainly, in bibliographical and documentary sources. It was concluded that extending the effects of immobility to management workers would be possible as protection for the family and the child with disabilities and not the parents themselves, since the legal instruments in force, which promulgate the National System for Integral Protection of the Boy, Girl and Adolescents before mentioned, give room for the possibility of having this protection.

Key words: Immobility; management workers; stability; organic labor law 
INTRODUCCIÓN

Los países subdesarrollados a nivel mundial en la mayoría de las organizaciones, no están al tanto de la importancia que la protección de los trabajadores y trabajadoras para alcanzar los objetivos previsto de la mejor manera y con la mayor productividad posible, lo que ha ocasionado graves daños humanos como materiales, interfiriendo a su vez con la producción y funcionamiento de la empresa; motivo por el cual hoy día se requiere de personas capacitadas que brinden la asesoría adecuada a las organizaciones para velar por un adecuado ambiente de trabajo el cual le permita a sus integrantes desarrollar sus actividades con el menor margen de riesgo posible y de esa manera obtener un eficaz rendimiento a nivel laboral.

Desde esa perspectiva, para lograr Protección de los trabajadores y trabajadoras, existe en la literatura respectiva, muchos enfoques o formas d abordar este tema, uno de ellos el de mejoramiento continuo. Este enfoque, más que una orientación es una estrategia y como tal constituye una serie de programas generales de acción y despliegue de recursos para lograr objetivos completos en todos los procesos en que se aplique. Múltiples son los modelos de mejora continua existentes en la realidad empresarial actual, la mayor parte de estos se asocian al mejoramiento de la calidad de productos o servicios pero de forma general sus pasos o etapas pueden ser aplicados a cualquier función o proceso empresarial que se desee perfeccionar.

Cabe destacar, que a pesar de las diferencias existentes en cuanto a nivel de complejidad, numero de pasos o etapas a aplicar pueden observarse un conjunto de puntos comunes entre los modelos para la protección de los trabajadores y trabajadoras referidos, los cambios de ritmo, de producción, los horarios, las tecnologías, aptitudes personales, y otros, generan una serie de condiciones que pueden afectar a la salud, todas estas premisas dan a considerar las condiciones de trabajo dentro de las organizaciones que conllevan a un conjunto de variables que definen la realización de una tarea en un entorno determinando la salud del trabajador en función de tres variables básicas como física, psicológica y social.

A pesar de su importancia, la situación de las condiciones de trabajo constituyen una barrera para la competitividad en cuanto a la productividad y calidad de servicio final, ya que de dicha condiciones depende el esfuerzo o empeño que el trabajador ejerza sobre su labor; hecho que les impide a algunas organizaciones posicionarse de mejor manera no solamente en los mercados locales y nacionales sino también en los internacionales. La prevención de los riesgos en el trabajo así como las medidas que se deben emprender para evitar los accidentes y las lesiones y prevenir las enfermedades que se pueden adquirir en el trabajo constituye un desafío de mayor importancia en las actuales circunstancias. En ese sentido, se requiere no sólo políticas específicas y de una fiscalización adecuada, sino también poner en marcha medidas prácticas y de bajo costo que permitan a los gerentes y supervisores soluciones sencillas y de fácil implementación.

De tal manera, los principios fundamentales para el establecimiento de buenas prácticas laborales en función de estrategias que mejoren las condiciones de trabajo, están reflejados en el mandato de la Organización Internacional del Trabajo (OIT) desde su creación en 1919 y se encuentran contenidos en sus convenios y recomendaciones sobre seguridad y salud, y condiciones de trabajo Sobre la base, de la actualidad en Venezuela la temática laboral ha tomado un papel significativo en el día a día de las organizaciones, a pesar de los grandes problemas que han surgido al tratar de adaptarse a los nuevos tiempos de 
globalización; se considera de gran importancia que las organizaciones posean un programa de seguridad laboral, para que de esta manera romper con el paradigma de la explotación de los trabajadores y trabajadoras y así velar por su salud; cuyo programa debe contener las normas idóneas para realizar cada operación eficaz y eficientemente para el control de accidente de trabajo.

Refiriéndonos concretamente al objeto de este artículo, es necesario señalar que, la Ley Orgánica de los Trabajadores, Trabajadoras y del Trabajo, publicada en Gaceta Oficial Extraordinaria № 6076 (LOTTT),de la República Bolivariana de Venezuela ha desarrollado en sus artículos 347 y ordinal 4 del artículo 420, según lo expresado en la exposición de motivos, la inamovilidad laboral extensiva a todos los trabajadores que no sean temporeros o de dirección y el derecho al trabajo y el deber de trabajar de las personas de acuerdo con sus capacidades y aptitudes, incluyendo a las personas con discapacidad.

De allí que, los Trabajadores de Dirección están excluidos de la aplicación de los supuestos del ordinal 4 del artículo 420 y 347 de la LOTTT, situación que permite indagar por qué no se hizo extensivo a estos trabajadores la protección de inamovilidad; para lo cual debe partirse de los requisitos de procedencia de la inamovilidad referida a los sujetos de derecho del trabajo y la legitimidad que debe ostentar quien pretenda hacer uso de ella.

Cabe destacar la importancia de este estudio, el cual persigue analizar la posibilidad de incluir y extender los efectos de la inamovilidad laboral a los trabajadores de dirección, que encuadren en los supuestos del artículo 347 y ordinal 4 del artículo 420 de la LOTTT, como medida de protección al hecho social trabajo y al derecho constitucional del trabajo y el deber de trabajar y por vía de consecuencia, la protección a la familia.

Tal como lo plantea Alfonzo-Guzmán, (2001) dentro del tema de investigación debe revisarse, lo establecido tanto en la doctrina como en la jurisprudencia con respecto a la estabilidad y a la inamovilidad, lo cual es una figura o subespecie que deriva de ésta, buscándose en principio entender y averiguar ¿qué es? Y ¿a quiénes se aplica?, cuestión de capital importancia a la hora de argumentar el por qué debería o no aplicarse dicha regulación al trabajador de dirección, quien en principio según lo establecido en la Ley del Trabajo, no goza de estabilidad, y por ende de inamovilidad, por tanto, en dicho estudio debe tomarse en consideración los principios jurídicos y sociales que rigen la realidad actual, y con la entrada en vigencia de un nuevo contrato social como lo fue la Constitución de la República Bolivariana de Venezuela (1999), ya que Venezuela se convirtió en un estado social de derecho y de justicia, donde se propugna la justicia y la paz social, el desarrollo y progresividad de los derechos sociales, que en este caso serían los derechos humanos, el derecho al trabajo y la protección a la familia como parte de éstos.

\section{Importancia del problema}

De acuerdo con García. (2012) en cuanto a que los trabajadores de dirección, son una categoría de trabajadores que siempre han estado excluidos del régimen de inamovilidad bien sea por Decreto o por ley, incluso; no se encuentran amparados por la estabilidad laboral. Los trabajadores y trabajadoras de dirección, se han caracterizado por estar inmersos en un régimen que sí bien no están dentro de los regímenes especiales, tampoco están dentro del régimen aplicable al contrato laboral común, los mismos pueden ser despedido sin que medie ninguna causa; los trabajadores y trabajadoras de dirección, son quienes intervienen en la toma de decisiones u orientaciones de la empresa, así como los que tiene el carácter de representantes del patrono frente a otros trabajadores o terceros y puede sustituirlo, en todo o en parte, en sus funciones. 
Desde este punto de vista, según Sainz (2013) es importante analizar los requisitos de procedencia de la protección de la Inamovilidad Laboral y la factibilidad de poder extender sus efectos a los trabajadores de dirección en caso que éstos tengan hijos niños, con alguna discapacidad o enfermedad que les impida valerse por sí mismo atendiendo a las leyes sustantivas que rigen la materia, y específicamente dentro de los supuestos establecidos en los artículos 347 y 420 Ordinal 4 de la LOTT, tomando en cuenta las características de este tipo de trabajadores y las implicaciones que ocasionaría en las relaciones de trabajo dentro de las cuales se desempeñan.

En opinión de Petit, (2009) Estos hechos significativos conllevan a estudiar la exclusión o inclusión, de los trabajadores de dirección de la aplicación de la inamovilidad laboral establecida en la Ley del Trabajo, de las Trabajadoras y Trabajadores; no obstante el carácter social, de protección e inclusión de esta nueva ley; razón por la cual resulta pertinente realizar un estudio jurídico laboral, sobre la extensión de inamovilidad especial a estos trabajadores, esta temática parte de una realidad especial y particular que caracteriza a este tipo de trabajadores, por cuanto los mismos son parte importante en la conducción y ejecutoriedad de los objetivos de la institución tanto pública como privada, donde presten servicios, y que su permanencia en ellas pudiera ocasionar o no conductas que contravengan las metas de la institución.

\section{MÉTODO}

El estudio se enmarcó dentro de la investigación documental, jurídica dogmática bajo un estudio monográfico, dado que se abordó un tema con sustento en los procesos de acopio de información, organización, análisis crítico y reflexivo, interpretación y síntesis de referencias y otros insumos pertinentes al tema seleccionado, por tanto se realizó un estudio teórico-reflexivo y analítico sobre situaciones prácticas y problemas en esta área con el fin de describirlos, identificar factores intervinientes o posibles causas y sus posibles vías para su solución.

Se tiene como fuente principal de la presente investigación los textos nacionales e internacionales. Se tienen como bases legales principales la Constitución de la República Bolivariana de Venezuela, la Ley Orgánica del Trabajo y su Reglamento.

A la confrontación de ese fenómeno con normas, valores y principios constitucionales se contrae esta investigación, y tanto la doctrina como la jurisprudencia aportarán los elementos indispensables para alcanzar con éxito los objetivos propuestos.

\section{RESULTADOS}

\section{Discusión}

En el marco de la investigación sobre la extensión de la inamovilidad contenida en el artículo 347 y 420 ordinal 4 de la Ley Orgánica del Trabajo a los trabajadores de dirección, como mecanismo de protección del estado a la permanencia en el trabajo, es importante destacar las actividades que realizan esta categoría de trabajadores, para definirlos como trabajadores de dirección, tomando en cuenta que prestan servicio de carácter laboral; no obstante, por su naturaleza, no gozan de algunos beneficios laborales.

En tal sentido, cabe preguntarse ¿cuáles fueron las características que presentan los trabajadores y las trabajadoras de dirección que lo diferencia como tal frente a otras categorías de trabajadores?

Los trabajadores y trabajadoras de dirección, se caracterizan como sujetos de la relación laboral, en las actividades realizadas que inciden en el destino de la unidad de trabajo donde laboran. 
En este sentido, considera García (2012) que no todo trabajador que en un momento dado tenga una función de representante del patrono frente a los demás trabajadores, o que intervenga en la ejecución de funciones puede ser calificado de trabajador de dirección, la Sala de Casación Social del Tribunal Supremo de Justicia, sostiene en sentencia de fecha 05 de agosto de $2011 \mathrm{~N}$ - 971 que la condición de empleado de dirección es de carácter excepcional y restringido, por lo que esta denominación únicamente se aplica a los altos ejecutivos o gerentes de las empresas, que participan en lo que se conoce como las grandes decisiones.

Es de advertir, por lo antes expuesto, que el trabajador de dirección constituye una categoría especial de trabajador, que conlleva su no inclusión dentro de los supuestos de inamovilidad establecidos en los artículos 420 ordinal 4 y 347, de la Ley Orgánica del Trabajo, las Trabajadoras y los Trabajadores.

Corresponde destacar que las normas en las cuales está expresamente excluido el trabajador de dirección, ellas son art. 87 LOTTT, los excluye directamente del procedimiento de estabilidad, también están excluidos del Decreto Presidencial $N^{\circ} 2.158$, mediante el cual se establece la inamovilidad laboral a favor de los trabajadores $y$ trabajadoras del sector privado y del sector público regidos por la Ley Orgánica del Trabajo las Trabajadoras y los Trabajadores, ya que el Ejecutivo Nacional Prorroga la Inamovilidad Laboral.

Ahora bien, para poder responder la pregunta ¿por qué no se incluyó a los trabajadores de dirección dentro de los supuestos establecidos en los artículos 420 ordinal 4 y 347 de la Ley Orgánica del Trabajo, los Trabajadores y las Trabajadoras?; hay dos conceptos básicos a definir y aclarar: Empleado de Dirección e Inamovilidad como sub especie de la estabilidad absoluta por cuanto en estos artículos, la protección establecida es de inamovilidad.
Por consiguiente, visto $\mathrm{y}$ analizado ¿quiénes son trabajadores de dirección?, ¿qué los caracteriza?, se puede determinar que en el caso del articulo 420 LOTTT, no incluye en efecto, al trabajador de dirección aun cuando nombra algunos casos de trabajadores (con fuero maternal, fuero sindical etc.) y el articulo 347 LOTTT, sólo habla de trabajadores en forma genérica, y ni los menciona ni los excluye, pero puede pensarse sin lugar a dudas que están fuera de esta regulación y la razón es que esta categoría de trabajadores están expresamente excluidos por el articulo 87 LOTTT, lo que lleva a pensar ¿Por qué para el legislador patrio los trabajadores de dirección no pueden gozar de protección laboral, inamovilidad o estabilidad?

La respuesta sería que, dada la particularidad de las funciones que cumplen esta categoría de trabajadores como lo son: realizar actos de disposición, actuar como representante legal del patrono frente a los trabajadores y a terceros sustituyéndolo en todo o en parte, remover al personal, tomar decisiones sobre el rumbo estratégico y comercial de la empresa, y cualesquiera otras actividades para la consecución de los fines productivos de la empresa tal como lo haría su propio dueño.

La protección de inamovilidad, establecida en los artículos aludidos anteriormente, está referida a la inamovilidad en forma permanente de la cual gozan la trabajadora o el trabajador que tenga uno o más hijos o hijas con alguna discapacidad o enfermedad que le impida o dificulte valerse por sí mismo; puede observarse que el legislador incorporó el artículo 347, dentro del Título VI De La Protección de la Familia en el Proceso Social del Trabajo y el artículo 420 ordinal 4ํㅜㄹ dentro del Título VII Del Derecho a la Participación Protagónica de los Trabajadores, Trabajadoras y sus Organizaciones Sociales, Capítulo I Del Fuero Sindical, Sección Novena Del Fuero Sindical o 
Inamovilidad Laboral, donde se establece los procedimientos y la clase de trabajadores amparados por ésta.

Ahora bien, con fines proteccionistas en fecha 05 de enero de 2007, fue promulgada la Ley para las Personas con Discapacidad, en atención al deber del Estado de proteger a los discapacitados siendo una ley cuyo objeto y contenido consiste en establecer las disposiciones que permitan el desarrollo integral de las personas con discapacidad dentro de la sociedad, la participación solidaria de ésta, la familia, los entes públicos nacionales, estadales y municipales; así como también los entes privados nacionales 0 internacionales y organizaciones sociales.

También se podría argumentar, la inclusión en aplicación del principio de igualdad, pero en este caso no aplica por cuanto el trabajador de dirección no está en igualdad de condiciones con sus pares trabajadores, por cuanto las funciones que realiza le permiten desarrollarse en un ámbito totalmente diferente a los demás y por cuanto goza de un conjunto de beneficios igualmente distintos a los demás trabajadores.

El supuesto establecido en los artículos 420 ordinal 4oㅗ y 347 de la Ley Orgánica del Trabajo, las trabajadoras y los rabajadores, se refiere a la protección especial de inamovilidad permanente de la que gozan los trabajadores con hijos con alguna discapacidad o enfermedad que les impida o dificulte valerse por sí mismo, siendo la causa de la protección la condición de éstos niños con capacidades diferentes que requieren cuidados especiales y a su vez están expuestos a ser marginados socialmente $\mathrm{y}$ por ende ostentan una situación vulnerable.

La Convención sobre los Derechos del Niño (CDN) adoptada en 1989, es el primer Tratado Internacional que incluye una referencia específica a la discapacidad; el artículo 2 se refiere a la no discriminación de los niños con discapacidad. Del mismo modo, existe el artículo 23, el cual señala que los Estados Partes reconocen que el niño mental o físicamente impedido deberá disfrutar de una vida plena y decente en condiciones que aseguren su dignidad, le permitan llegar a valerse por sí mismo y faciliten la participación activa del menor en la comunidad.

No obstante, se escapa de lo desarrollado en la norma una categoría de trabajadores, tal es el caso de los trabajadores de dirección con hijos con condiciones especiales o discapacidad, que si bien cumplen funciones gerenciales, por su condición de trabajadores de dirección no están exento de una situación humana "como lo es tener hijos con discapacidad", hecho que le puede ocurrir a cualquier individuo independientemente de su condición social o posición económica.

Por cuanto se trata de proteger a la familia como tal y no al trabajador, es entendido que ante la existencia de un niño o niña con alguna discapacidad y que no puede valerse por sí mismo, por razones de índole social, de salud, de interés superior al niño, por mandato constitucional y en virtud de normas internacionales como lo es la Convención de los Derechos del niño adoptada y abierta la firma y ratificada por la Asamblea General en su Resolución 44/25 de fecha 20 de noviembre de 1989 y entró en vigencia el 02 de septiembre de 1990, se pudiese proteger a los padres a fin de garantizarle al niño o niña una mejor calidad de vida a pesar de su discapacidad.

Lo anterior, justifica la inclusión de los trabajadores de dirección en el marco de protección de inamovilidad prevista en los artículos 420 ordinal 4o, y 347 de la Ley Orgánica del Trabajo, las trabajadoras y los trabajadores.

Se vislumbra una posición contraria, sosteniendo la necesidad de la inclusión de ese tipo de trabajadores, dentro de los supuestos arriba mencionados, dada la 
Doctrina de Protección Internacional y el Sistema Rector Nacional para la Protección Integral de los Niños, Niñas y Adolescentes, contenida en la Convención Sobre los Derechos del Niño, Constitución de la República Bolivariana de Venezuela y la Ley Orgánica para la Protección de Niños, Niñas y Adolescentes, cuyo alcance proteccionista debe extenderse a todos los niños; es decir el derecho de uno debe ser igual al de todos, haciendo la salvedad, que la inamovilidad que se estudia, debe ser otorgada no por la figura del trabajador, sino por la condición de discapacidad de los hijos niños, niñas y adolescentes de estos trabajadores.

\section{CONCLUSIONES}

En este análisis se consideró la posibilidad de incorporar dentro de éste esquema de protección de inamovilidad a los trabajadores de dirección, los cuales históricamente han estado excluidos de cualquier protección en materia de estabilidad, toda vez que los mismos personifican la figura del patrono y esa ha sido la justificación a tal proceder. Sin embargo, lo que se pretende es proteger a los niños, niñas y adolescentes que se encuentren en situación vulnerable por un estado de discapacidad que le impida valerse por sí mismo, independientemente del trabajo que desempeñen sus padres, esto significa que la protección se debe brindar al niño considerando su interés superior, por cuanto al garantizar la permanencia laboral de sus padres, directamente se protegen a los niños o adolescentes en situaciones especiales de discapacidad.

Por tanto, extender los efectos de la inamovilidad a los trabajadores de dirección sería posible como protección a la familia y al niño con discapacidad y no propiamente a los padres, ya que los instrumentos legales vigentes, que promulgan el Sistema Rector
Nacional para la Protección Integral del Niño, Niña y Adolescentes antes enunciados, dan cabida a la posibilidad de contar con esta protección.

En el desarrollo de la historia desde el surgimiento de la relación obrero - patronal el hombre ha buscado mecanismos de defensa de sus derechos como trabajador. El sentido primordial del Derecho del Trabajo, es "la protección al trabajador", principio que desde sus inicios históricos ha tendido a proteger a la parte más débil de la relación bilateral, el trabajador, en ese contexto la necesidad de incorporar la Defensa Publica de Derechos Laborales para trabajadores que perciban bajos ingresos económicos, es una propuesta coincidente con la esencia del Derecho del Trabajo.

El conocimiento de sus derechos por parte del trabajador es de fundamental importancia, para hacerlos valer ante el empleador, sin embargo su conocimiento de nada sirve si el trabajador no tiene los medios para hacerlos valer.

Una contribución importante para el reconocimiento de los derechos de los trabajadores, es la incorporación de la defensa pública en materia laboral, ya que de este modo los trabajadores, podrán enfrentarse a los empleadores con el patrocinio de un abogado proporcionado por el Estado, permitiendo igualdad en la relación obrero patronal.

La mayoría de los trabajadores y extrabajadores se sienten dañados en sus derechos laborales por parte de sus empleadores, sin embargo, la mayoría no realiza reclamo alguno ante el Ministerio de Trabajo, y mucho menos ante Juez en materia laboral las mismas llegan a ser víctimas silenciosas.

Todos los trabajadores y ex trabajadores encuestados, en el trabajo de campo de la presente investigación, considera que la falta 
de dinero es un obstáculo para hacer valer sus derechos.

En Latinoamérica, existe una tendencia generalizada de incorporar dentro de la Defensa Publica, la materia laboral.

\section{REFERENCIAS}

Alfonzo-Guzmán, J. (2001). Nueva Didáctica del Derecho del Trabajo, $12^{\circ}$ edición adaptada a la Constitución de 1999 y a la Ley Orgánica del Trabajo y su Reglamento, Editorial Melvin C.A., Caracas

Constitución de la República Bolivariana de Venezuela (1999). Gaceta Oficial № 36.860, 30/12/1999.

Convención Sobre los Derechos Del Niño, Adoptada y abierta a la firma y ratificación por la Asamblea General en su Resolución $44 / 25$, de (20 de noviembre de1989) Entrada en vigor: 2 de septiembre de 1990, de conformidad con el artículo 49
García, J. (2012). Sustantivo Laboral en Venezuela. Caracas. Venezuela

Ley Orgánica del Trabajo. Gaceta Oficial Extraordinaria № 6.076 del 07/05/2012.

Ley para las Personas con Discapacidad. Gaceta Oficial Número 38.598. Caracas - 5 de Enero de 2007

Márquez, V. (2002). Estudios sobre la relación de trabajo. Universidad Católica Andrés Bello, Caracas

Petit, F. (2009). La estabilidad laboral y sus Procedimientos en la Legislación Venezolana. Caracas- Venezuela. Ediciones Libertad

Sainz, C. (2013) Lineamientos Prácticos de la Nueva Ley Orgánica del Trabajo, los Trabajadores y las Trabajadoras (LOTTT). Caracas Venezuela. 